Bio - grafía. Escritos sobre la Biología y su Enseñanza. ISSN 2027-1034

Edición Extraordinaria. p.p. 509 - 517

Memorias del IX Encuentro Nacional de Experiencias en Enseñanza de la Biología y la Educación Ambiental. IV Congreso Nacional de Investigación en Enseñanza de la Biología.

\title{
CONCEPCIONES DE LA VIDA DE LOS NIÑOS DE GRADO QUINTO DE UNA INSTITUCIÓN EDUCATIVA DE BOGOTÁ VICTIMAS DEL CONFLICTO: UN APORTE A LA ENSEÑANZA DE LABIOLOGÍA
}

\author{
CONCEPTIONS OF THE LIFE OF CHILDREN OF GRADE FIFTH OF AN \\ EDUCATIONAL INSTITUTION OF BOGOTÁ VICTIMS OF THE CONFLICT: A \\ CONTRIBUTION TO THE TEACHING OF BIOLOGY
}

\author{
Jean Pierre Diaz ${ }^{1}$ \\ Jhojan Guillermo Botache ${ }^{2}$
}

\section{RESUMEN}

El presente trabajo muestra los resultados de una propuesta investigativa desarrollada durante el trabajo de grado en el marco del proceso de formación de la Universidad Pedagógica Nacional en el año 2016-2017, trabajo realizado en la ciudad de Bogotá en una Institución Educativa.

La investigación se realiza con niños víctimas del conflicto, cuyos nombres e identidades se protegieron al igual que su ubicación, por efectos de seguridad, por ello no se nombra el Colegio al cual pertenecen.

Esta investigación se realiza bajo los planteamientos de la Hermenéutica-Interpretativa, la cual hace énfasis en el comprender e interpretar realidades, por lo cual se tiene en cuenta diferentes métodos de recolección de datos como entrevistas, dibujos artísticos e historias de vida, métodos que ayudan a comprender las realidades de vida y experiencias que han tenido que pasar los niños víctimas del conflicto.

Los resultados están organizados en 3 categorías principales: Concepciones de vida, desde sus historias de vida y ¿qué es la vida? A partir de los resultados se realizan orientaciones pedagógicas, teniendo en cuenta los planteamientos de la autopoiesis, reflexión, emoción, lenguaje, buscando el sentido de vida, biofilia, sentir-pensar y subsistencia humana.

Se concluye que, la educación es aquel proceso de transformación de las conductas para la convivencia, donde los niños víctimas tengan la posibilidad de experimentarlas en la vida misma. Este proceso debe llevar consigo la continua reflexión que la educación, el maestro y la enseñanza de la Biología deben asegurar.

\footnotetext{
${ }^{1}$ Estudiante décimo semestre de Licenciatura en Biología, Universidad Pedagógica Nacional. Correo institucionaldbi_jdiaz503@pedagogica.edu.co

2 Estudiante décimo semestre de Licenciatura en Biología, Universidad Pedagógica Nacional. Correo institucional: dbi_jbotache659@pedagogica.edu.co
} 
Bio - grafía. Escritos sobre la Biología y su Enseñanza. ISSN 2027-1034

Edición Extraordinaria. p.p. 509 - 517

Memorias del IX Encuentro Nacional de Experiencias en Enseñanza de la Biología y la

Educación Ambiental. IV Congreso Nacional de Investigación en Enseñanza de la Biología.

PALABRAS CLAVE: Vida, vivo, víctimas del conflicto, enseñanza de la biología, emoción.

\begin{abstract}
ABST ACT
The present work shows the results of a research proposal developed during the work of degree within the framework of the training process of the National Pedagogical University in the year 2016-2017, work done in the city of Bogotá in an Educational Institution.

The investigation is carried out with children victims of the conflict, whose names and identities were protected as well as their location, for security purposes, for that reason does not name the College to which they belong.

This research is carried out under the Hermeneutic-Interpretative approach, which emphasizes understanding and interpreting realities, which takes into account different methods of data collection such as interviews, art drawings and life stories, methods that help to understand the realities of life and experiences that the children victims of the conflict have had to go through.

The results are organized into 3 main categories: Conceptions of life, from their life histories and what is life? Based on the results, pedagogical orientations are made, taking into account the approaches of autopoiesis, reflection, emotion, and language, seeking the meaning of life, biophilia, feeling-thinking and human subsistence.

It is concluded that, education is that process of transformation of behaviors for coexistence, where child victims have the possibility of experiencing them in life itself. This process must carry with it the continuous reflection that education, the teacher and the teaching of Biology must ensure.
\end{abstract}

KEY WORDS: Life, live, victims of conflict, teaching of biology, emotion.

\title{
INTRODUCCIÓN
}

El presente trabajo investigativo surge del interés de comprender las concepciones de los niños víctimas del conflicto, debido a la coyuntura del país y el proceso de paz que se adelanta por parte del Gobierno Nacional y las Fuerzas Armadas Revolucionarias de Colombia (FARC). Siguiendo con lo anterior se dio a la tarea de buscar la población niños víctimas del conflicto en la ciudad de Bogotá, Colombia en Instituciones Educativas o Centros de Atención a Víctimas. 
Bio - grafía. Escritos sobre la Biología y su Enseñanza. ISSN 2027-1034

Edición Extraordinaria. p.p. 509 - 517

Memorias del IX Encuentro Nacional de Experiencias en Enseñanza de la Biología y la

Educación Ambiental. IV Congreso Nacional de Investigación en Enseñanza de la Biología.

Posteriormente, se encuentra la población de niños víctimas donde se inicia el trabajo con seis (6) niñas y cuatro (4) niños oriundos de diferentes partes del Territorio Nacional y que por efectos del conflicto se han tenido que desplazar a la ciudad de Bogotá.

Es por eso que hablar de la vida en el mundo contemporáneo se torna bastante complejo, debido a la pluralidad de definiciones que existen, ya que las diferentes áreas del conocimiento se han encargado de definirla, conceptualizarla e interpretarla de diferentes formas de acuerdo a su propio objeto de estudio.

Desde la enseñanza de la biología es importante tener en cuenta las concepciones de los niños víctimas del conflicto, pues se considera que desde las concepciones de vida pueden llegar a ser fundamentales en la comprensión de fenómenos y problemáticas desde un ámbito biológico y social.

La metodología de investigación es cualitativa con enfoque hermenéutico interpretativo, la cual se retoma por el alto valor que le da a la experiencia, al dialogo y la comprensión de realidades vital a la hora de interpretar y reconocer las historias de vida de los niños víctimas. Se tienen en cuenta historias de vida, entrevistas y actividades donde realizan dibujos de sus lugares de procedencia. Teniendo en cuenta los aportes metodológicos, se determinan ocho fases de trabajo, donde se plantean actividades en relación a las concepciones de vida de los niños víctimas del conflicto, desde sus historias de vida y entrevistas que giraron a través de la pregunta ¿qué es la vida?

En los resultados arrojados se resaltan los sentires, emociones y concepciones de los niños en relación a la vida. Concepciones que se analizan, se comprenden y se interpretan con ayuda de matrices de categorización. Teniendo al amor como la principal emoción para acoger a los niños y poder enseñar biología, además de ello desde el amor se establece una relación de reconocimiento por el otro.

Finalmente se concluye al amor como principal emoción donde se legitima al otro en la convivencia, donde los niños víctimas del conflicto a través de la emoción reconocen al otro desde la interacción y el continuo reconocimiento de sus vidas. Se hace necesario incluir la emoción en la enseñanza de la biología, pues desde allí se puede generar una enseñanza donde se motive al estudiante a ver la vida y la biología desde el contexto, usando todos los sentidos para percibirla y a través de estos procesos crear valores de conservación y cuidado por todas las formas de vida.

\section{METODOLOGÍA.}

\section{HERMENEUTICA-INTERPRET ATIVA}

"el ser del hombre reside en comprender"

Gadamer (1999)

Para este ejercicio investigativo lo "hermenéutico- interpretativo" es retomado por el énfasis en el comprender realidades y por el alto valor que le da a la experiencia, dos 
Bio - grafía. Escritos sobre la Biología y su Enseñanza. ISSN 2027-1034

Edición Extraordinaria. p.p. 509 - 517

Memorias del IX Encuentro Nacional de Experiencias en Enseñanza de la Biología y la Educación Ambiental. IV Congreso Nacional de Investigación en Enseñanza de la Biología.

elementos importantes para el presente trabajo y para su desarrollo metodológico. De igual forma la experiencia como elemento fundante del proceso hermenéutico, ya que ésta incorpora inevitablemente la dimensión temporal y con ello el reconocimiento histórico de la experiencia. Lo expuesto permite captar la importancia a los elementos socioculturales que otorga al proceso de interpretación.

Gadamer (1900-2002) y otros autores enfatizan que "la hermenéutica debe ser entendida como el arte del entendimiento, a partir del diálogo" factor importante en el desarrollo de la investigación, desde el dialogo y la conversación se inicia un proceso de construcción de la historia narrada por los niños víctimas del conflicto.

Por otra parte, Giannini (1998) citado en Cárcamo (2005) que " imaginar es interpretar comprensivamente y comprender será el mecanismo para percibir la intención ajena" y en esa misma vía Gadamer (1999) sostiene que "el ser del hombre reside en comprender" para lo anterior se requiere de un sujeto consciente y con capacidad de reconocer su historicidad. Es aquí, desde la comprensión donde junto con la experiencia, el dialogo e inicia a reconstruir la historia de cad niño que ha sido golpeado por el conflicto de Colombia. Es por ello que la hermenéutica hace grandes aportes a la hora de analizar y comprender cada palabra y sentimiento expresado por los niños víctimas.

\section{FASES DEL TRABAJO}

De este modo se proponen diferentes métodos de recolección de datos como entrevistas, dibujos artísticos, historias de vida, los cuales ayudan a obtener información de primera mano, que facilitan la elaboración de resultados, visibilizando la voz de los niños víctimas del conflicto acerca de sus concepciones acerca de la vida.

FASE I Contextualización: Se identificaron las características esenciales de la población en cuestión, se realizó la contextualización pertinente del lugar, de la institución y de las problemáticas particulares de los sujetos.

FASE II Socialización del propósito del trabajo: Se socializó con los niños el propósito del trabajo investigativo y la incidencia que ellos iban a tener dentro del mismo

FASE III: Caracterizar concepciones Se socializa la primera actividad a realizar, la cual consiste en hacer dibujos, donde representen sus lugares de origen, y el lugar actual donde viven.

FASE IV COMPRENSIÓN: Con respecto a los resultados de la anterior fase, se hace necesario indagar ciertos elementos o fenómenos que no quedaban claros en los dibujos realizados por los niños, para ello se hace un registro de audio preguntando el ¿por qué? de ciertos dibujos.

FASE V HISTORIAS DE VIDA: Se generó otra estrategia, las historias de vida, aquí los niños escribían sus lugares de procedencia, experiencias que han tenido que vivir, la forma de cómo llegaron a la capital, escriben en relación a su familia, de quienes la conforman y como es la relación con ellos. 
Bio - grafía. Escritos sobre la Biología y su Enseñanza. ISSN 2027-1034

Edición Extraordinaria. p.p. 509 - 517

Memorias del IX Encuentro Nacional de Experiencias en Enseñanza de la Biología y la Educación Ambiental. IV Congreso Nacional de Investigación en Enseñanza de la Biología.

FASE VI ¿Qué es la vida?: Se realiza entrevistas de forma grupal acerca de una pregunta ¿qué es la vida? Con el objetivo de generar debate entre los niños y poder evidenciar diversidades de opiniones y concepciones acerca de esta concepción.

FASE VII CATEGORIZACIÓN: Se leyeron, transcribieron y se categorizaron los trabajos y entrevistas de los niños sobre sus historias de vida y las concepciones que presentan en relación a la vida.

FASE VIII: PROPUESTA EDUCATIVA PARA LA FORMACIÒN DE LOS NIÑOS VICTIMAS DEL CONFLICTO: A partir de los resultados de investigación, nace la necesidad de plantear una propuesta educativa donde se particularice la situación de los niños víctimas del conflicto.

En cuanto a la Enseñanza de la Biología se hace un aporte significativo, en el sentido que se retoma una población que no ha sido tenida en cuenta en investigaciones dentro de la línea, como los niños víctimas del conflicto, donde las concepciones de vida de éstos niños víctimas deben ser tenidas en cuenta y a portar a la Enseñanza de la Biología.

\section{RESULT ADOS.}

\section{Concepciones de la vida:}

Se evidencia que la mayoría de los niños víctimas del conflicto de la Institución Educativa, son oriundos de diferentes partes del Territorio Nacional, siendo procedentes de Antioquía, Boyacá, Tumaco, Magangué Bolívar, Santa Marta, Cartagena, San José del Guaviare, Santander. Cabe resaltar que la mayoría de los niños vivieron en las periferias o en el sector rural de dichas ciudades.

"yo vengo de Tumaco, el lugar que más me gustaba de allí es el mar y la playa porque jugaba mucho con mis amigos y mi familia" [sic]

... "yo vengo de Ayapel Córdoba lo que más recuerdo y extraño es mi familia y mi casa, allí éramos felices" [sic]

... "yo vengo de Magangué extraño el mar, como lo dibujé y las chalupas, los animales que veía en el mar" [sic]

Se observa la conexión que hay entre sus vidas y el contexto de donde provienen, la conexión que presenta los niños con el mar y su familia y con los lugares que más recuerda, debido a que son significativos para ellos.

Los niños recuerdan sus lugares de procedencia por la interacción que tuvieron con los animales, pues muchos de ellos vivieron en el campo, en fincas y tuvieron contacto por mucho tiempo con ellos, tanto así que algunos de ellos los consideran como familia:

"yo vengo de Santander, allí lo que más me gustaba eran las vacas, toros, marranos porque jugaba con ellos y son importantes para mi porque crecícon ellos"[sic] 
Bio - grafía. Escritos sobre la Biología y su Enseñanza. ISSN 2027-1034

Edición Extraordinaria. p.p. 509 - 517

Memorias del IX Encuentro Nacional de Experiencias en Enseñanza de la Biología y la Educación Ambiental. IV Congreso Nacional de Investigación en Enseñanza de la Biología.

..."Lo que más me gustaba de Cartagena aparte del mar eran los peces que podía ver y diferentes animales que me gustaba, un día vi un caballito de mar" [sic]

\section{Historias de vida.}

Los niños han tenido que vivir separaciones, las cuales les ha afectado de gran manera en su vida, pues no han tenido la posibilidad de compartir tiempo, ni experiencias con sus padres:

... "yo vivo con mis abuelos paternos, no vivo con mis papás porque hubo un problema en el barrio de nosotros, entonces mi mamá está en la cárcel por algo que ella no hizo, y la declararon culpable... una vez estábamos en el 2013, entonces mi hemana el novio le pegó, entonces ella bajó golpeada con mi mamá, mi papá estaba tomando entonces el cogió una pistola, el disparó, la bala traspasó la puerta y se lo pego en la espalda y lo mató" [sic]

Estas fueron algunas de las situaciones que viven nuestros estudiantes, conviven con mil dificultades, con recuerdos en sus mentes que no son adecuados para niños, niños que han visto de cerca matar, robar en sus barrios:

"yo lo que me acuerdo de mi vida es que fue muy dura porque mi papá trabajaba en una zorra y mi mamá trabajaba en un zorro, bueno después mataron a mi tía y después mataron a mi tío gonito, un día mi papá se agarró con un señor y unos tíos le pegaron un tiro" [sic]

Desde sus lugares de origen han tenido que percibir, sentir, vivir y experimentar el conflicto, los vestigios de una guerra que se ha perpetuado y que han afectado a centenares de personas, especialmente a los niños, dejándolos en una situación de vulnerabilidad...

"yo vivía con mi abuelita, hace dos años me fui para Tumaco y yo estaba muy pequeño y comencé a estudiar me acuerdo que hubo una balacera en Tumaco que mataron a varias personas, donde yo vivía era muy peligroso, se veía la guerra entre ellos" [sic]

De igual forma a uno de los niños que han vivido en estas hostilidades han tenido que ver a sus familiares tras las rejas, pues han sido parte de la guerrilla como lo expresa una niña de tan solo 11 años de edad:

... "yo cuando nací yo viví en Tarasá con mi mamá mi papá y mi hermano y yo, a mi papá lo cogió la tomba y lo metieron a la cárcel porque era guerrillero, y ahora vive con otra novia" [sic]

... "Mi papá está en la cárcel por algo privado, es muy triste porque no puedo jugar con él, no lo puedo ver porque no me dejan entrar a verlo, espero que se encuentre bien" [sic]

\section{¿Qué es la vida?}

La vida y su resistencia a una sola definición, está ligada a la gran diversidad y a la complejidad de cada una de las definiciones que se le otorgue a la vida, es por eso que los niños la siguen definiendo así: 
Bio - grafía. Escritos sobre la Biología y su Enseñanza. ISSN 2027-1034

Edición Extraordinaria. p.p. 509 - 517

Memorias del IX Encuentro Nacional de Experiencias en Enseñanza de la Biología y la

Educación Ambiental. IV Congreso Nacional de Investigación en Enseñanza de la Biología.

"la vida es ser feliz, compartir con los amigos cosas importantes, y estar con la familia" [sic]

... "la vida ha sido difícil la muerte de mi primo, (llanto) lo recuerdo mucho porque jugábamos mucho, era mi mejor amigo" [sic]

... "profe yo recuerdo la muerte de mi abuelita eso me marcó la vida" [sic]

Pero a pesar de que ha sido injusta también han tenido momentos importantes y que han marcado su vida.

..."a mí lo que me marcó mi vida fue el hecho de nacer, solo con eso" [sic]

... "la vida es dura, pero hay que ser felices con nuestras familias, y que cuando tú te mueras, puedas pensar que tuviste una bonita vida, sin miedos" [sic]

Se propone varias estrategias desde la didáctica y la pedagogía, las cuales van dirigidas a maestros tanto en ejercicio como en formación. En concordancia con lo anterior, se plantea que es necesario enseñar biología a los niños víctimas del conflicto desde la emoción teniendo como base fundamental el amor que en palabras de Maturana (1995) "el amor, es la emoción donde el otro tiene una existencia legítima, donde no se le niega, sino que se le acepta como un otro válido. $Y$ es desde ahí que se puede construir una vida en sociedad"

De igual forma se propone que la enseñanza de la biología y desde el papel del maestro en la educación deben propiciar espacios de reflexión continua donde se enriquezca la capacidad de acción de los niños, la autoconstrucción personal basados en los planteamientos de la autopoiesis, desde la conversación y la biología del amor. Se destaca la importancia de la búsqueda de sentido de vida en los niños víctimas del conflicto, pues desde allí se les puede devolver la esperanza y la emoción de vivir, se hace necesario e indispensable, debido a su condición de vulnerabilidad.

Estos planteamientos se hacen en relación a los aportes realizados por Viktor Frankl (1991), quien fuera participe del horror en los campos de concentración nazi. A partir de los planteamientos de Frankl, se debe aprender que la vida, por más dura y difícil siempre tendrá sentido, depende de la actitud con que la afrontemos las adversidades y la capacidad de amar como emoción fundamental para sobrevivir en un momento de holocausto que se presente en la vida. Se pudo entender que desde el mismo sufrimiento se puede encontrar el sentido, el cual muy probablemente nos convierta en los héroes de nuestra propia vida.

Por último se propone a la biofilia y el sentir pensar como una estrategia para el maestro. Desde la biofilia, se asume como el amor que tiene el ser humano por la vida y lo vivo, lo cual permite que se dé un filiación emocional importante, donde este proc eso lleva a los sujetos a tener una identidad y una realización personal (Kellert, 1999, pp. 42-69). Así mismo el sentir pensar, se asume como aquel proceso mediado por la razón y el corazón, donde la razón está fundamentada desde la emoción. 
Bio - grafía. Escritos sobre la Biología y su Enseñanza. ISSN 2027-1034

Edición Extraordinaria. p.p. 509 - 517

Memorias del IX Encuentro Nacional de Experiencias en Enseñanza de la Biología y la

Educación Ambiental. IV Congreso Nacional de Investigación en Enseñanza de la Biología.

\section{CONCLUSIONES:}

Desde el trabajo investigativo, se considera la educación como aquel proceso de transformación de las conductas para la convivencia, donde los niños víctimas del conflicto tengan la posibilidad de experimentarlas en la vida misma, junto con el maestro, compañeros y familiares, es decir donde tenga un proceso de acompañamiento. Lo anterior debe ser acompañado por un proceso de reflexión continua que la misma educación, Enseñanza de la Biología y desde el maestro deben asegurar.

Los niños víctimas del conflicto, se mostraron como son, niños con sentires, emociones y pensamientos, niños que desde el encuentro con los autores del trabajo mostraron la disposición de expresar acontecimientos de su vida, dejando ver sus emociones y sentimientos de tristeza, exponiendo contextos particulares en el conflicto de Colombia. Es por ello que el ámbito educativo debe retomar el contexto de los niños, las experiencias y realidades como aporte a la enseñanza, pues se evidencia que hay una ruptura, puesto que por el afán de la escuela de culturizar, de reproducir contenidos, de preparar a los estudiantes para el mundo laboral, se olvidan y desconocen las realidades particulares, se olvidan de los contextos de los niños, de sus vivencias y de las concepciones de vida que tienen desde sus mismas experiencias. Hay que comenzar a repensar la educación donde oriente al estudiante mediante al aprendizaje a transformarse desde la reflexión.

Se establece el amor como principal emoción donde se legitima al otro en la convivencia, donde los niños víctimas del conflicto a través de la emoción reconocen al otro desde la interacción y el continuo reconocimiento de sus vidas. Se hace necesario incluir la emoción en la enseñanza de la biología, pues desde allí se puede generar una enseñanza donde se motive al estudiante a ver la vida y la biología desde el contexto, usando todos los sentidos para percibirla y a través de estos procesos crear valores de conservación y cuidado por todas las formas de vida.

\section{BIBLIOGRAFÍA:}

Abad, J. (2013) Basta Ya! Colombia: Memoria de Guerra y Dignidad. Resumen. Centro de Memoria paz y reconciliación. Bogotá. Colombia

Alvarado, S \& Tapia, L (2012) Las Escuelas como Territorios de Paz. Ed Clacso. Bogotá Colombia. Recuperado de http://biblioteca.clacso.edu.ar/Colombia/alianza-cindeumz/20130313112059/LasEscuelascomoTerritoriosdePaz.pdf

Aponte, D (2009) Guerra y Violencias en Colombia. Herramientas e Interpretaciones. Ed Pontificia Universidad Javeriana. Bogotá Colombia.

Buitrago, S \& Fernández, A (2013). "La experiencia de vivir la vida desde la participación en la enseñanza de la biología”. Revista biografía. Universidad Pedagógica Nacional.: 
Bio - grafía. Escritos sobre la Biología y su Enseñanza. ISSN 2027-1034

Edición Extraordinaria. p.p. 509 - 517

Memorias del IX Encuentro Nacional de Experiencias en Enseñanza de la Biología y la Educación Ambiental. IV Congreso Nacional de Investigación en Enseñanza de la Biología.

Bogotá. Recuperado de http://revistas.pedagogica.edu.co/index.php/biografia/article/viewFile/2422/2264

Cándida, M (2001) Educar y Aprender en la Biología del Amor. Brasilia. Brasil. Recuperado de http://www.ub.edu/sentipensar/pdf/educar_y_aprender.pdf

Castaño, N (2015). Polisemia de las concepciones acerca de la vida desde una mirada occidental. Universidad Pedagógica Nacional. Express estudio gráfico y digital: Bogotá

Castaño, N. (2015)."Concepciones de vida, cosmogonía Uitoto, enseñanza de la Biología y diversidad cultural. Aspectos metodológicos”. Researchgate: Bogotá. 